\title{
A New Medical Device to Provide Independent Ventilation to Two Subjects Using a Single Ventilator: Evaluation in Lung-Healthy Pigs
}

\author{
Ignacio Lugones $\mathbb{D}^{\mathrm{D}},{ }^{1}$ Roberto Orofino Giambastiani, ${ }^{1}$ Oscar Robledo, ${ }^{2}$ Martín Marcos, ${ }^{2}$ \\ Javier Mouly, ${ }^{2}$ Agustín Gallo, ${ }^{1}$ Verónica Laulhé, ${ }^{1}$ and María Fernanda Biancolini ${ }^{1}{ }^{1}$ \\ ${ }^{1}$ Hospital General de Niños "Pedro de Elizalde", Ciudad Autónoma de Buenos Aires, Buenos Aires, Argentina \\ ${ }^{2}$ Facultad de Ciencias Veterinarias, Universidad Nacional de La Plata, Provincia de Buenos Aires, La Plata, Argentina \\ Correspondence should be addressed to Ignacio Lugones; cardiocongenitas@gmail.com
}

Received 20 July 2020; Revised 25 October 2020; Accepted 18 December 2020; Published 30 December 2020

Academic Editor: Basavana B. Goudra

Copyright (c) 2020 Ignacio Lugones et al. This is an open access article distributed under the Creative Commons Attribution License, which permits unrestricted use, distribution, and reproduction in any medium, provided the original work is properly cited.

\begin{abstract}
Background. The global crisis situation caused by SARS-CoV-2 has created an explosive demand for ventilators, which cannot be met even in developed countries. Designing a simple and inexpensive device with the ability to increase the number of patients that can be connected to existing ventilators would have a major impact on the number of lives that could be saved. We conducted a study to determine whether two pigs with significant differences in size and weight could be ventilated simultaneously using a single ventilator connected to a new medical device called DuplicARß. Methods. Six pigs (median weight $12 \mathrm{~kg}, \mathrm{range} 9-25 \mathrm{~kg}$ ) were connected in pairs to a single ventilator using the new device for 6 hours. Both the ventilator and the device were manipulated throughout the experiment according to the needs of each animal. Tidal volume and positive end-expiratory pressure were individually controlled with the device. Primary and secondary outcome variables were defined to assess ventilation and hemodynamics in all animals throughout the experiment. Results. Median difference in weight between the animals of each pair was 67\% (range: 11-108). All animals could be successfully oxygenated and ventilated for 6 hours through manipulation of the ventilator and the DuplicAR $\circledast$ device, despite significant discrepancies in body size and weight. $\mathrm{Mean} \mathrm{PaCO}_{2}$ in arterial blood was $42.1 \pm 4.4 \mathrm{mmHg}$, mean $\mathrm{PaO}_{2}$ was $162.8 \pm 46.8 \mathrm{mmHg}$, and mean oxygen saturation was $98 \pm 1.3 \%$. End-tidal $\mathrm{CO}_{2}$ values showed no statistically significant difference among subjects of each pair. Mean difference in arterial $\mathrm{PaCO}_{2}$ measured at the same time in both animals of each pair was $4.8 \pm 3 \mathrm{mmHg}$, reflecting the ability of the device to ventilate each animal according to its particular requirements. Independent management of PEEP was achieved by manipulation of the device controllers. Conclusion. It is possible to ventilate two lung-healthy animals with a single ventilator according to each one's needs through manipulation of both

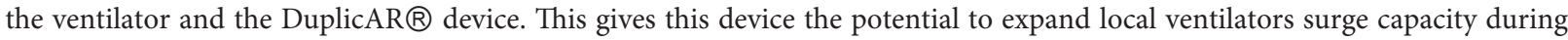
disasters or pandemics until emergency supplies can be delivered from central stockpiles.
\end{abstract}

\section{Background}

As a consequence of sudden major catastrophes such as terrorism attacks, natural disasters, massive accidents, or epidemic outbreaks, health systems have focused on the need for simultaneous medical attention for a large number of victims [1-3].

Depending on the nature and suddenness of the disaster, many hospital supplies, such as ventilators, may become insufficient to face the demand. Although health system resources can be immediately redirected for equipment provision, there is a variable time period between the initial need of supplies and the moment they can actually be provided. During this interval, many patients could die $[4,5]$.

The world crisis triggered by the SARS-CoV-2 pandemic has created an explosive demand for mechanical ventilators. Such demand could not be met even in developed countries 
[6]. Healthcare teams are pushed to decide whether to withdraw or withhold ventilators for some patients and use them for other patients who have better chances of survival. This scenario requires the preparation of a triage system to better allocate the critical care resources available to maximize the benefit for the greatest number of people [7], which could create a possible legal liability [8].

The design of a simple and inexpensive device that allows simultaneous and independent ventilation of two patients with only one ventilator could have a significant impact on the number of lives that could be saved.

The objective of this study is to test, in a lung-healthy porcine model, a device designed to ventilate two subjects with the same ventilator, called DuplicAR $®$ (patent application submitted).

\section{Materials and Methods}

2.1. Protocol Design. This is a translational research study with an experimental prospective and controlled protocol design, approved by the Institutional Animal Care and Use Committee of the School of Veterinary Sciences, National University of La Plata, Argentina, and the Ethics Committee of the General Children's Hospital of Buenos Aires “Dr. Pedro de Elizalde," Argentina.

2.2. Animal Model. Six healthy pigs, with variable weights (median weight $12 \mathrm{~kg}$, range 9-25 kg), coming from the local farm of the School of Veterinary Sciences of the National University of La Plata, previously dewormed and free of contagious infectious diseases, were included in the study. Animal handling was carried out following an update of the Guide for the Care and Use of Laboratory Animals of the National Research Council US Committee [9].

2.3. Preparation and Anesthesia. After intramuscular sedation with midazolam $0.5 \mathrm{mg} / \mathrm{kg}$ (Richmond Vet Pharma, Buenos Aires, Argentina) and ketamine $15 \mathrm{mg} / \mathrm{kg}$ (Richmond Vet Pharma, Buenos Aires, Argentina), each animal was connected to pulse oximetry and cardiac leads monitoring and placed in a prone position to allow ear vein cannulation. A surgical plane of anesthesia was obtained with intravenous propofol at $8 \mathrm{mg} / \mathrm{kg}$ in an induction dose and $15 \mathrm{mg} / \mathrm{kg} / \mathrm{h}$ in continuous infusion (Richmond Vet Pharma, Buenos Aires, Argentina). The animals were intubated orotracheally and ventilated manually with $100 \%$ oxygen throughout the setup phase. The tibial artery was cannulated with a 20-gauge single lumen catheter, used for blood pressure measurements and arterial blood gas sampling. Mean arterial blood pressure (MAP), heart rate (HR), and pulse oximetry $\left(\mathrm{SpO}_{2}\right)$ were recorded using multiparameter monitors (PM-9000, Shenzhen Mindray BioMedical Electronics, Shenzhen, China).

At this time, the animals were connected to the ventilator after an intravenous dose of vecuronium bromide $0.1 \mathrm{mg} / \mathrm{kg}$ (Scott-Cassará, Buenos Aires, Argentina).
2.4. The DuplicAR@ Device. The animals were connected in pairs, each pair to a single ventilator using the new device.

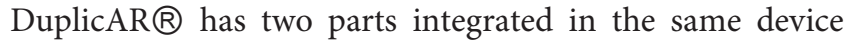
(Figure 1).

The inspiratory part of the device connects the inspiratory port of the ventilator to each animal's inspiratory limb. It has ball valves that regulate flow (and therefore tidal volume $\left(V_{\mathrm{T}}\right)$ and peak pressure) and one-way valves that (1) allow for independent management of the two circuits and (2) avoid cross-contamination.

The expiratory limb of each animal connects to the expiratory part of DuplicAR@, which in turn attaches to the expiratory port of the ventilator. It has one-way valves to prevent cross-ventilation and keep the patients' circuits isolated from each other. It also has a positive end-expiratory pressure (PEEP) valve, which is embedded in a small box and interposed in the expiratory limb of the patient that needs higher PEEP (Figure 1(b)). The amount of pressure configured on the PEEP valve will be added in that particular subject to the PEEP configured in the ventilator, in order to achieve the target PEEP for that subject. The remaining subject, with no PEEP valve interposed in the expiratory limb, will have the PEEP that was configured on the ventilator.

2.5. Ventilation. Volume assist-control ventilation was adjusted on the ventilator to achieve a $V_{\mathrm{T}}$ of $10-15 \mathrm{ml} / \mathrm{kg}$ of combined pig weight, respiratory rate between 16 and 22 breaths/min, inspiratory time between 0.75 and 1.3 seconds, fraction of inspired oxygen of 0.3 , and PEEP of $2 \mathrm{cmH}_{2} 0$. The $V_{\mathrm{T}}$ controllers of the DuplicAR® device were manipulated to ensure adequate thoracic expansion and symmetrical pulmonary auscultation in both animals, according to their respective needs.

$\mathrm{SpO}_{2}$ and end-tidal $\mathrm{CO}_{2}$ (etCO 2 ) were monitored continuously and provided important information during the initial ventilation setup. The airway pressure-time waveform of each animal was obtained using a regular arterial/venous pressure transducer (filled with air) connected to the $Y$-piece located at the confluence of the inspiratory and expiratory limbs of each subject and displayed in $\mathrm{cmH}_{2} \mathrm{O}$ in the screen of the multiparameter monitor.

The animals were ventilated for 6 hours. A different ventilator was used in each experiment to test their compatibility with DuplicAR@: (a) Newport Breeze E-150 (Newport Medical Instruments, CA, USA), (b) Avea (CareFusion, CA, USA), and (c) Maquet Servo I (Maquet Critical Care AB, SE). Throughout the procedure, adjustments on the ventilator and the device were made based on clinical evaluation of each animal, cardiac monitoring, $\mathrm{SpO}_{2}$, capnography, and arterial blood gas parameters. In order to specifically assess the ability of the device to ventilate two different subjects independently, we focused on monitoring $\mathrm{PaCO} 2$, establishing the goal of achieving a normal value of this variable in arterial blood.

After 6 hours of combined ventilation, the different controllers of this novel device were manipulated in order to simulate the need for differential PEEP among animals. We 


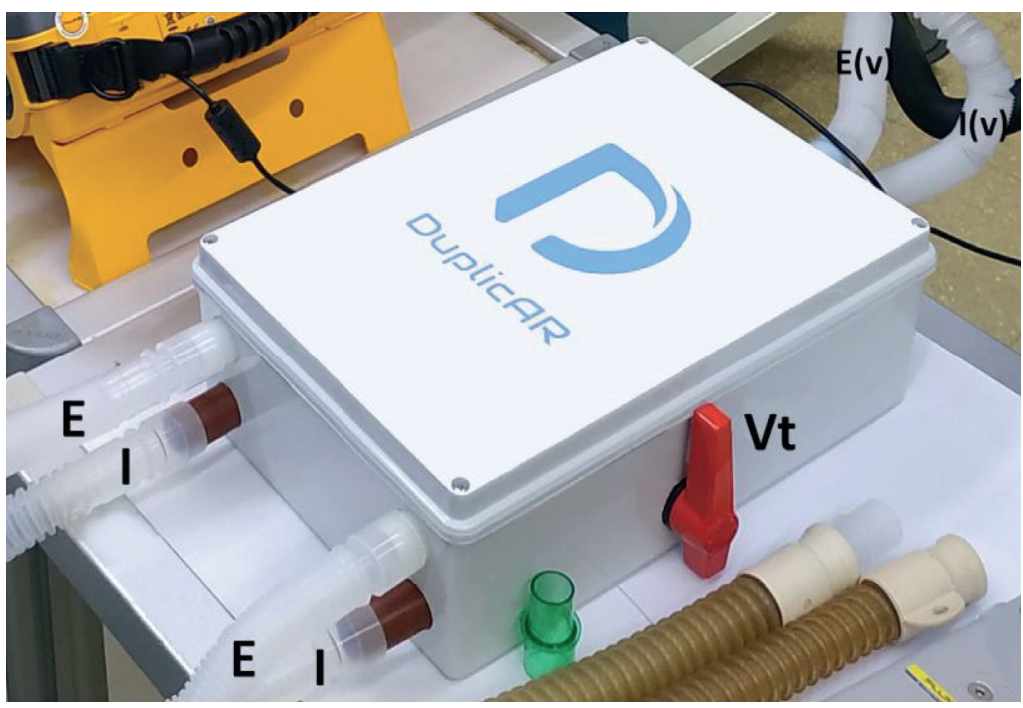

(a)

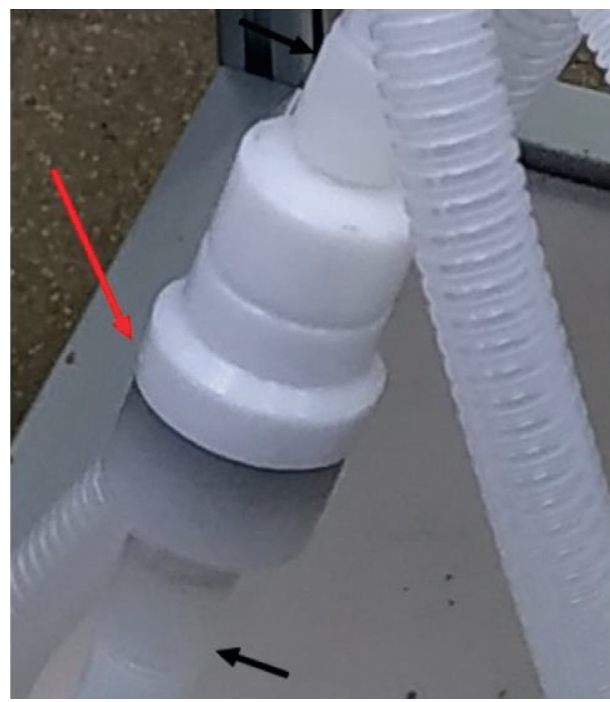

(b)

Figure 1: The DuplicAR® device: (a) the device with its connections; (b) the PEEP valve controller. E, expiratory limb of each subject; E(v), expiratory limb connected to the ventilator; I, inspiratory limb of each subject; I(v), inspiratory limb connected to the ventilator; Vt, tidal volume/peak-pressure controller; red arrow, PEEP controller; black arrows, expiratory limb.

performed selective increases in the PEEP in one animal of each pair through manipulation of the respective controller, in order to achieve a PEEP of 10 and $15 \mathrm{cmH}_{2} \mathrm{O}$ sequentially, while maintaining a PEEP of $2 \mathrm{cmH}_{2} \mathrm{O}$ in the other animal.

2.6. Data Collection and Analysis. Primary outcome variables reflecting ventilation and oxygenation performance were $\mathrm{PaCO}_{2}$, etCO, $\mathrm{PaO}_{2}$, and $\mathrm{SpO}_{2}$. Secondary outcome variables were also defined: $\mathrm{pH}, \mathrm{MAP}, \mathrm{HR}$, and serum lactate. Baseline $(t=0)$ was defined as the moment when both subjects were connected to the ventilator with the DuplicAR $®$ device. Thereafter, vital signs were recorded every 15 minutes during the first hour, every 30 minutes during hours 2 and 3 , and every 60 minutes between hours 4 and 6. Arterial blood samples were obtained at $t=30 \mathrm{~min}$, $t=2 \mathrm{hr}$, and $t=5 \mathrm{hr}$ and examined in a blood gas/electrolyte analyzer (Gem Premier 3000, Instrumentation Laboratory, Lexington, MA).

Primary and secondary outcome variables are reported as mean \pm standard deviation and calculated using Microsoft Excel version X (Microsoft, Redmond, Washington, USA). Student's $t$-test was used for paired samples.

\section{Results}

Median difference in weight between the animals in each pair was $67 \%$, ranging from $11 \%$ in the first experience to $108 \%$ in the last one.

Mean $\mathrm{PaCO}_{2}$ in arterial blood was $42.1 \pm 4.4 \mathrm{mmHg}$ (Figure 2). No statistically significant difference was found in this variable between subjects connected to the same ventilator at any time of the experiment. Mean difference in $\mathrm{PaCO}_{2}$, measured at the same time in both animals, in each pair was $4.8 \pm 3 \mathrm{mmHg}$. An adequate oxygenation was maintained throughout the experiment in all animals, as reflected by a mean $\mathrm{PaO}_{2}$ of $162.8 \pm 46.8 \mathrm{mmHg}$, while mean $\mathrm{SpO}_{2}$ was $98 \pm 1.3 \%$.

$\mathrm{EtCO}_{2}$ values were registered hourly and compared between animals. No statistically significant difference was found between subjects connected to the same ventilator (AB pair: $p=0.65$; C-D pair: $p=0.64$; E-F pair: $p=0.73$ ).

All subjects remained hemodynamically stable during the procedure. MAP was $79 \pm 17 \mathrm{mmHg}$, while mean $\mathrm{HR}$ was $115 \pm 27$ beats/min. Mean $\mathrm{pH}$ was $7.46 \pm 0.05$, and serum lactate values were below $1.31 \mathrm{mmol} / \mathrm{l}$ in all animals at all times (Table 1).

Manipulation of the PEEP controllers in one animal of each pair (animals $\mathrm{A}, \mathrm{C}$, and $\mathrm{E}$ ) allowed a selective increase of the PEEP in that animal to 10 and $15 \mathrm{~cm} \mathrm{H}_{2} \mathrm{O}$ successively, while PEEP in the other animal remained around $2 \mathrm{cmH}_{2} \mathrm{O}$ (as set in the baseline configuration of the ventilator) (Figure 3).

\section{Discussion}

The idea of ventilating multiple patients with a single mechanical ventilator was described by Neyman and Irvin in 2006 [10]. In this study, four lung simulators were ventilated with a single ventilator, using 3-way connectors to have 4 inspiratory limbs and 4 expiratory limbs. In 2008, Paladino and collaborators tested the proposed method in an animal model, concluding that it was feasible to simultaneously ventilate four sheep with a single ventilator for 12 hours [11]. Afterwards, Smith and Brown reported simultaneous ventilation of two healthy human beings with a single ventilator and emphasized on the importance of matching both subjects such that both could tolerate the chosen pressures and achieve an acceptable $V_{\mathrm{T}}$ [12]. However, in 2012, Branson and Rubinson concluded that it was not possible to 


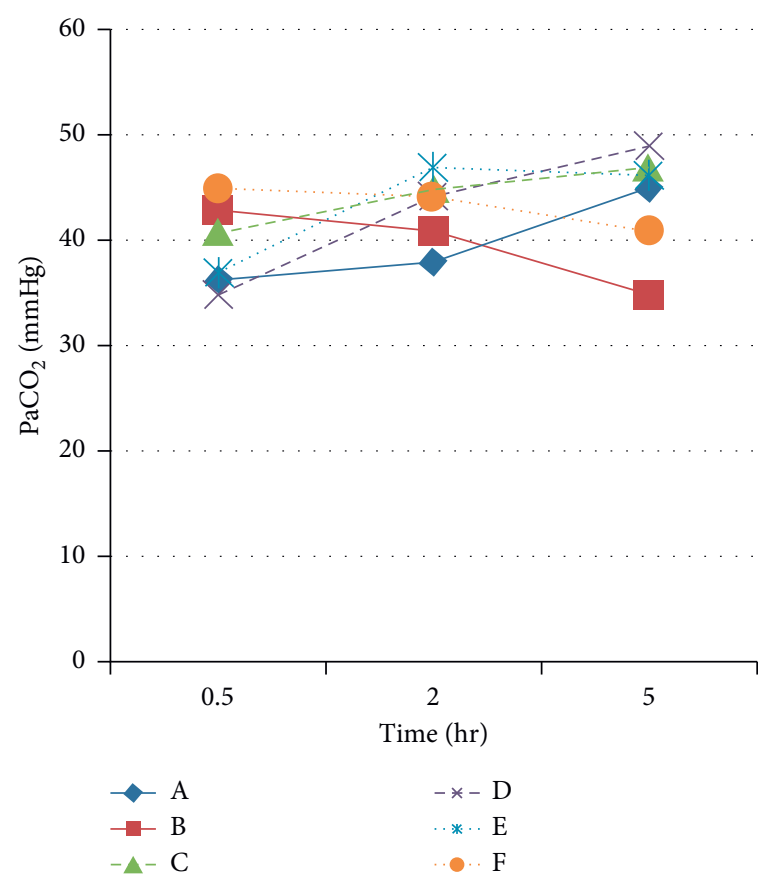

(a)

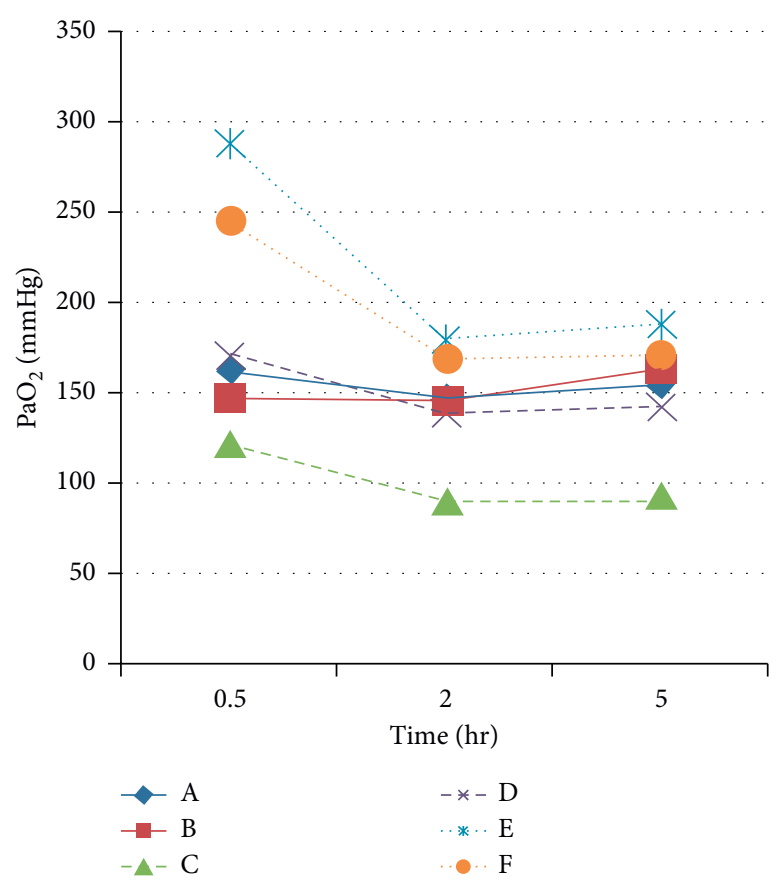

(b)

Figure 2: (a) $\mathrm{PaCO}_{2}$ and (b) $\mathrm{PaO}_{2}$ registered at $t=30$ minutes, $2 \mathrm{hr}$, and $5 \mathrm{hr}$ in all subjects.

TABLE 1: Primary and secondary outcome variables in the six animals throughout the experiment.

\begin{tabular}{|c|c|c|c|c|c|c|c|c|c|c|c|c|}
\hline & \multicolumn{4}{|c|}{ Pair 1} & \multicolumn{4}{|c|}{ Pair 2} & \multicolumn{4}{|c|}{ Pair 3} \\
\hline & \multicolumn{2}{|c|}{$t=30^{\prime}$} & \multicolumn{2}{|c|}{$t=5 \mathrm{hr}$} & \multicolumn{2}{|c|}{$t=30^{\prime}$} & \multicolumn{2}{|c|}{$t=5 \mathrm{hr}$} & \multicolumn{2}{|c|}{$t=30^{\prime}$} & \multicolumn{2}{|c|}{$t=5 \mathrm{hr}$} \\
\hline & A & B & A & B & $\mathrm{C}$ & $\mathrm{D}$ & $\mathrm{C}$ & $\mathrm{D}$ & E & F & E & $\mathrm{F}$ \\
\hline Weight & 10 & 9 & & & 20 & 12 & & & 25 & 12 & & \\
\hline $\mathrm{pH}$ & 7.46 & 7.4 & 7.37 & 7.46 & 7.54 & 7.58 & 7.47 & 7.45 & 7.57 & 7.48 & 7.46 & 7.5 \\
\hline $\mathrm{PaCO}_{2}$ & 36 & 43 & 45 & 35 & 41 & 35 & 47 & 49 & 37 & 45 & 46 & 41 \\
\hline $\mathrm{PaO}_{2}$ & 162 & 148 & 156 & 164 & 123 & 171 & 94 & 143 & 289 & 246 & 189 & 172 \\
\hline $\mathrm{SO}_{2}$ & 99 & 99 & 99 & 100 & 96 & 99 & 95 & 99 & 99 & 100 & 96 & 100 \\
\hline $\mathrm{HR}$ & 116 & 120 & 101 & 128 & 108 & 101 & 124 & 90 & 119 & 90 & 158 & 106 \\
\hline MAP & 80 & 70 & 68 & 80 & 138 & 89 & 93 & 70 & 90 & 88 & 103 & 92 \\
\hline Lactate & 1.24 & 0.96 & 1.29 & 0.87 & 0.5 & 0.9 & 0.5 & 0.8 & 0.7 & 0.8 & 0.6 & 1 \\
\hline
\end{tabular}

control the $V_{\mathrm{T}}$ that each patient would receive and the disparity in this volume was due to the variability in the airway compliance between patients [13]. Also, the pressure control mode exacerbated the disparity more than the volume-control mode.

During the COVID-19 pandemic, several healthcare teams have considered multiple ventilation (using 3-ways connectors in the inspiratory and expiratory ports of the ventilator) as an attractive and rapidly available alternative. However, this strategy has many limitations that make it an unsafe option. In fact, it has been totally discouraged by some of the most important scientific societies in the world [14]. The main drawback of this mode is its inability to manage the ventilatory parameters independently for each subject, especially the $V_{\mathrm{T}}$, the peak pressure, and the PEEP, both in the initial connection and over time. The lack of a device that distributes pressure and flow according to the needs of each subject implies that the subjects must meet similar criteria in terms of weight, clinical condition, and pulmonary compliance to become candidates to be ventilated simultaneously [15]. On the other hand, there are important challenges in monitoring ventilation and setting alarms, and it is necessary to consider that a sudden event occurring in one patient (e.g., disconnection, pneumothorax, and obstruction of the endotracheal tube) will impact on the other.

As part of the COVID-19 surge response plan, many groups have explored alternative strategies to overcome the problems of ventilator sharing [16]. At the beginning of the pandemic, our group developed a device called DuplicAR $\mathbb{R}$ as part of the efforts to face the disease in our country. In this pilot study, we show that it is possible to ventilate two lunghealthy subjects connected simultaneously to one ventilator and this novel device, according to their particular needs. Our first prototype was able to accomplish its main purpose, which was to achieve individual management of the $V_{\mathrm{T}}$ (or 


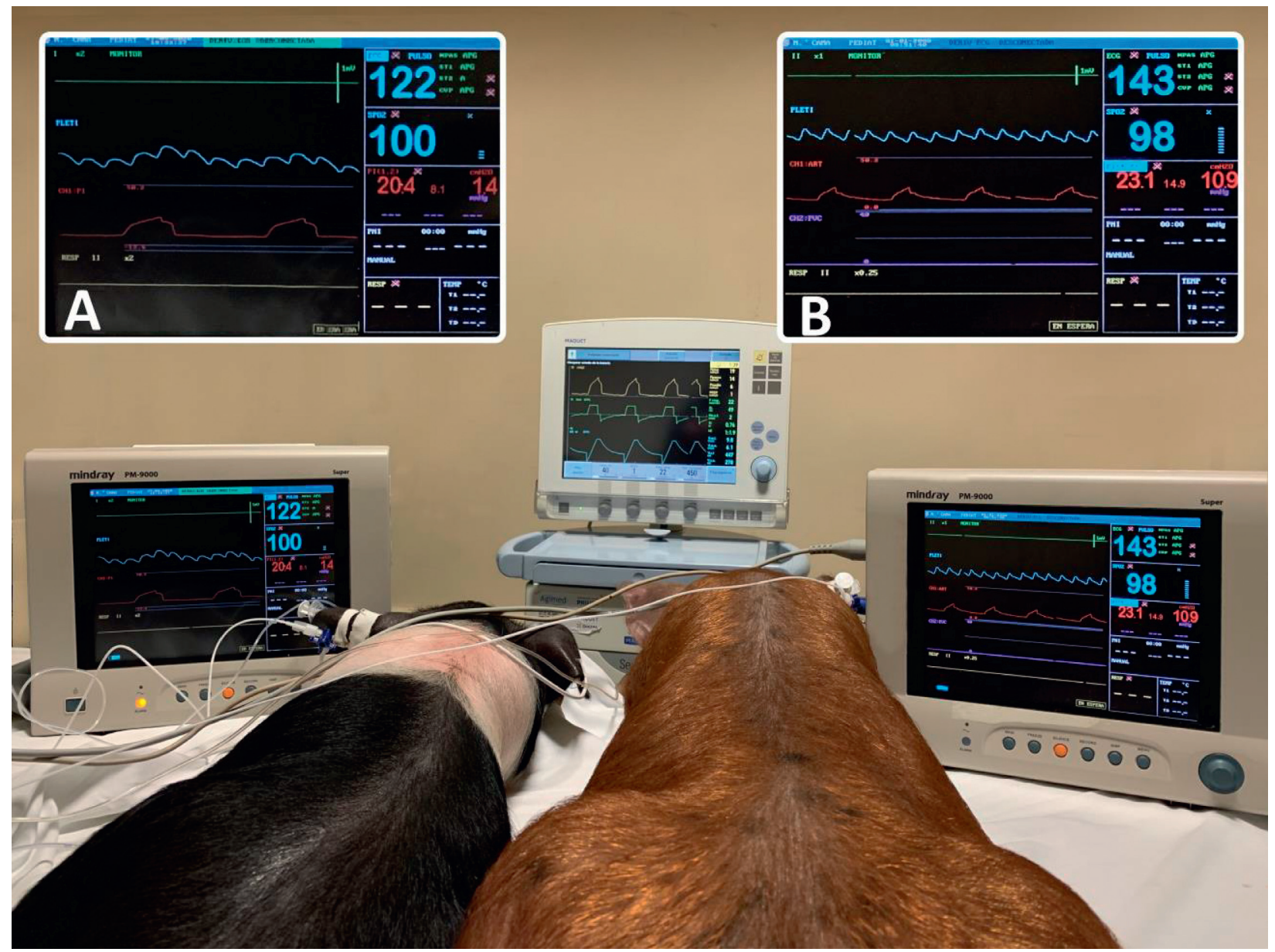

FIGURE 3: Two animals, weighing $12 \mathrm{~kg}$ (left) and $25 \mathrm{~kg}$ (right), are shown being ventilated simultaneously with a single ventilator and the DuplicAR $®$ device. The pressure-time curve of each animal is displayed in $\mathrm{cmH}_{2} \mathrm{O}$ in each multiparameter monitor (in detail in (a) and (b)). In (a), the peak pressure is $20.4 \mathrm{cmH}_{2} \mathrm{O}$, while the PEEP is $1.4 \mathrm{cmH}_{2} 0$. In (b), the peak pressure is $23.1 \mathrm{cmH}_{2} 0$, and the $\mathrm{PEEP}$ is $10.9 \mathrm{cmH}_{2} 0$.

peak pressure) and the PEEP in response to the requirements of each subject over time. All the animals were successfully ventilated and oxygenated for 6 hours and were hemodynamically stable all along the experiment. The targeted values of $\mathrm{PaCO}_{2}$ and etCO ${ }_{2}$, the main endpoints that reflected ventilation, remained within expected ranges in all cases, and most importantly, there were no significant differences in these variables between animals connected to the same ventilator despite significant discrepancies in body size and weight. This is the reason why we prefer to call this strategy "combined ventilation" rather than "multiple ventilation," as mutual interactions between the involved subjects are considered and individual needs are attended in order to ventilate them adequately. Regarding ventilation mode, pressure-controlled is probably safer than volumecontrolled ventilation, as the maldistribution of the $V_{\mathrm{T}}$ in the latter can cause an excessively high $V_{\mathrm{T}}$ to be delivered to one individual, which can result in volutrauma and worse situations, including barotrauma.

This early-stage prototype is under continuous improvement. At this moment, the pressure-time curve can be clearly displayed in each subject's multiparameter monitor using a pressure transducer and setting the monitor to measure in $\mathrm{cmH}_{2} \mathrm{O}$. This waveform allows for precise monitoring of peak pressure, plateau pressure and PEEP, the evaluation of the adequacy of the inspiratory flow, and the presence of auto PEEP, dynamic hyperinflation, or circuit leaks. The final version of the device will include direct realtime measurement of all variables of each subject and electronic closed loop control of peak pressure and PEEP of each individual through a human-machine interface.

It is important to note that this device is conceived to function only as a bridge to other alternatives in the context of disaster surge, when health systems are overwhelmed, and there are not as many ventilators as needed. To face these catastrophic events, this device can be easily stored and may provide fast availability of ventilation alternatives. As evidenced by this experiment, DuplicAR $®$ is compatible with the three different ventilators that were used and would probably adapt well to other models.

Specific recommendations for optimal use of this device are as follows: (a) subjects need to be appropriately sedated and paralyzed to prevent patient-ventilator interaction; (b) individual airway pressure measurement should be provided in multiparameter monitors; (b) pressure limit and alarms must be carefully set to avoid excessive peak pressure; (c) 
closed aspiration circuits should be ensured to prevent contamination and need for disconnection; (d) filters must be used in both inspiratory and expiratory limbs; and (e) capnography and respiratory mechanics monitoring are highly recommended for each patient.

4.1. Study Limitations. This is just a proof-of-concept experiment, in which we only demonstrate that two subjects can be ventilated independently with one ventilator using

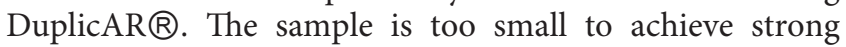
statistically significant conclusions.

The device allows independent manipulation of $V_{\mathrm{T}}$ (or peak pressure) and PEEP, but cannot control each subject's fraction of inspired oxygen, respiratory rate, and inspiratory time. Besides, this first prototype does not display (in the device itself) the curves and values of the different variables of both subjects nor does it have incorporated alarms.

A detailed evaluation of the performance of this device has been carried out both in a computational model and in the respiratory laboratory with two lung simulators, measuring all the relevant variables in different scenarios. Both experiments are being reported at the time this article is being written. The device has not yet been tested in subjects with lung injury, which is an important limitation.

Finally, the device must be tested in a pressure-controlled ventilation mode in an animal model. This mode is probably the safest to ventilate simultaneously two patients with the same ventilator [17].

\section{Conclusion}

It is possible to ventilate two lung-healthy animal subjects with the same ventilator using DuplicAR $\AA$ to regulate the $V_{\mathrm{T}}$ and the PEEP independently. This strategy might evolve into a life-saving bridge alternative to palliate the consequences of the sudden shortage of ventilators during catastrophic events.

\section{Data Availability}

The data used to support the findings of this study are available from the corresponding author upon request.

\section{Conflicts of Interest}

The authors declare that they have no conflicts of interest.

\section{References}

[1] D. D. Sommer, J. A. Fisher, V. Ramcharan, S. Marshall, and D. M Vidic, "Improvised automatic lung ventilation for unanticipated emergencies," Critical Care Medicine, vol. 22, no. 4, pp. 705-709, 1994.

[2] M. D. Christian, A. V. Devereaux, J. R. Dichter, L. Rubinson, and N. Kissoon, "Introduction and executive summary," Chest, vol. 146, no. 4, pp. 8S-34S, 2014.

[3] J. A. Cordova-Villalobos, A. E. Macias, M. Hernandez-Avila et al., "The 2009 pandemic in Mexico: experience and lessons regarding national preparedness policies for seasonal and epidemic influenza," Gaceta Medica de Mexico, vol. 153, pp. 102-110, 2017.

[4] E. L. Daugherty Biddison, R. Faden, H. S. Gwon et al., "Too many Patients A framework to Guide statewide allocation of scarce mechanical ventilation during disasters," Chest, vol. 155, no. 4, pp. 848-854, 2019.

[5] E. J. Emanuel, G. Persad, R. Upshur et al., "Fair allocation of scarce medical resources in the time of covid-19," New England Journal of Medicine, vol. 382, no. 21, p. 2049, 2020.

[6] M. L. Ranney, V. Griffeth, and A. K. Jha, "Critical supply shortages - the need for ventilators and personal protective equipment during the covid-19 pandemic," New England Journal of Medicine, vol. 382, no. 18, p. e41, 2020.

[7] R. C. Maves, J. Downar, J. R. Dichter et al., "Triage of scarce critical care resources in COVID-19 an implementation Guide for regional allocation," Chest, vol. 158, no. 1, pp. 212-225, 2020.

[8] I. G. Cohen, A. M. Crespo, and D. B. White, "Potential legal liability for withdrawing or withholding ventilators during COVID-19," JAMA, vol. 323, no. 19, pp. 1901-1902, 2020.

[9] National Academies Press, Guide for the Care and Use of Laboratory Animals, National Academies Press (US), Washington (DC), The National Academies Collection: Reports funded by, 8th edition, 2011.

[10] G. Neyman and C. B. Irvin, "A single ventilator for multiple simulated patients to meet disaster surge," Academic Emergency Medicine, vol. 13, no. 11, pp. 1246-1249, 2006.

[11] L. Paladino, M. Silverberg, J. G. Charchaflieh et al., "Increasing ventilator surge capacity in disasters: ventilation of four adulthuman-sized sheep on a single ventilator with a modified circuit,” Resuscitation, vol. 77, no. 1, pp. 121-126, 2008.

[12] R. Smith and J. M. Brown, "Simultaneous ventilation of two healthy subjects with a single ventilator," Resuscitation, vol. 80, no. 9, p. 1087, 2009.

[13] R. D. Branson, T. C. Blakeman, B. R. Robinson, and J. A. Johannigman, "Use of a single ventilator to support 4 patients: laboratory evaluation of a limited concept," Respiratory Care, vol. 57, no. 3, pp. 399-403, 2012.

[14] 2020 The society of critical care medicine (SCCM), American association for respiratory care (AARC), American society of anesthesiologists (ASA), anesthesia patient safety foundation (APSF), American association of critical-care nurses (AACN), and American college of chest physicians (CHEST). Joint Statement on Multiple Patients Per Ventilator SCCM, AARC, ASA, APSF, AACN, and CHEST Share Unified Message.

[15] J. R. Beitler, A. M. Mittel, R. Kallet et al., "Ventilator sharing during an acute shortage caused by the COVID-19 pandemic," American Journal of Respiratory and Critical Care Medicine, vol. 202, no. 4, pp. 600-604, 2020.

[16] A. L. Clarke, A. F. Stephens, S. Liao, T. J. Byrne, and S. D. Gregory, "Coping with COVID -19: ventilator splitting with differential driving pressures using standard hospital equipment," Anaesthesia, vol. 75, no. 7, pp. 872-880, 2020.

[17] J. Herrmann, A. Fonseca da Cruz, M. L. Hawley, R. D. Branson, and D. W. Kaczka, "Shared ventilation in the era of COVID-19: a theoretical consideration of the dangers and potential solutions," Respiratory Care, vol. 65, no. 7, pp. 932-945, 2020. 